und Monosemierung des Zugangs zu einem Thema beitragen möchte, die zudem verbunden ist mit einer hohen intertextuellen Fehlerreproduktion.

Richtig interessant werden digital humanities da, wo sie im weiteren Kontext einer »Theorie der digitalen Gesellschaft « (so A. Nassehi unter dem Haupttitel »Muster «, München 2019) und wissenssoziologischen Diskurstheorie (L.C. Martino) betrachtet werden. Dann geht es um die regelhafte Ordnung, welche Gesellschaft konstituiert wird und sich als Muster zeigt, indem Merkmale definiert und korreliert werden. Genau diese Zählbarkeit des Sozialen und Rekombinierbarkeit bestimmter Merkmale in Datenmengen ist das, was Digitalität meint. Die Merkmale allerdings müssen in gesellschaftlichen und wissenschaftlichen Diskursen erst definiert werden, denn nicht jedes Merkmal ist schon eine relevante Kategorie (meine Sockenfarbe ist vermutlich weniger relevant als meine Konfession). Insofern ist eine Selbstbeobachtung des Systems Gesellschaft immer involviert in diese komplexitätsreduzierende Digitalisierung, die auf sie folgen kann.

\title{
Das Wissenschaftliche Bibellexikon im Internet
}

WiBiLex. Das wissenschaftliche Bibellexikon im Internet, hg.v. Stefan Alkier/Michaela Bauks/ Klaus Koenen, 2007ff., online: https://www.bibelwissenschaft.de/wibilex (12.06.2020).

\section{Altes Testament}

Mathias Litzenburger

Das wissenschaftliche Bibellexikon im Internet (WiBiLex) ist ein Projekt der Deutschen Bibelgesellschaft und wird von M. Bauks und K. Koenen (Altes Testament) sowie von S. Alkier (Neues Testament) herausgegeben, wobei es zahlreiche weitere Fachherausgeber*innen und knapp 600 Autor*innen einzelner Artikel gibt. Das Lexikon entsteht seit 2007, umfasst derzeit etwa 1900 Artikel und wird laufend um neue Artikel erweitert (derzeit - Mitte April 2020 - scheint es einen leichten quantitativen Schwerpunkt auf alttestamentlichen Artikeln zu geben). Zudem können auch bereits vorhandene Lexikonartikel durch Fachautor*innen weiter aktualisiert werden: Der Artikel »Bileam (AT)« von E. Gaß wurde ursprünglich im Dezember 2007 erstellt, jedoch sind unterhalb des Artikels zwei weitere Versionen von 2018 und $2019 \mathrm{zu}$ finden. So ist es möglich, dass Artikel im Sinne neuerer Forschungsdiskurse und -ergebnisse überarbeitet und die jeweiligen Literaturverzeichnisse aktualisiert werden. Durch die gute fachliche Redaktion bietet das kostenfrei nutzbare WiBiLex verlässliche und fundierte Informationen zu einer großen Fülle bibelwissenschaftlicher Themen, so dass die einzelnen Artikel - anders als manch andere Internetquellen - zitierfähig sind. 
Im WiBiLex kann auf unterschiedliche Weise recherchiert werden. So befindet sich auf der Startseite eine alphabetische Liste aller Stichwörter, die man wahlweise scrollend durchsuchen oder aber durch einen Suchbegriff gezielter filtern kann. Die Nutzung von Suchbegriffen im Stichwortverzeichnis dürfte die effizienteste Recherchemöglichkeit sein, da beispielsweise bei der Eingabe des Schlagwortes »Mose « neben dem zentralen Artikel » Mose« auch weitere Artikel mit dem Begriff im Namen angezeigt werden (z.B. »Himmelfahrt des Mose«, aber auch - sachlich nicht zugehörig [s.u.] - »Almosen«). Die Schlagwörter führen dann zu den jeweiligen Artikeln; es muss also nur einmalig eine neue Seite geladen werden. Dieser Hinweis mag seltsam anmuten, ist aber vor dem Hintergrund ungewöhnlich langer Ladezeiten einzelner Artikel von nicht zu unterschätzender Bedeutung für das Gelingen einer schnellen Suche.

Die auffallende Langsamkeit scheint unabhängig vom Internetanbieter, von der Signalstärke des konkreten WLAN-Netzes und von dem benutzten Endgerät zu sein. Gerade bei umfangreicherer Recherche kann diese geringe Geschwindigkeit im Seitenaufbau - besonders im Vergleich zur sonstigen, heutzutage üblichen Internetgeschwindigkeit - durchaus störend wirken.

Über die weiter oben auf der Startseite befindliche Suchfunktion »Direkt zum Artikel« kann man einen konkreten Artikel direkt aufrufen, was jedoch mit Schwierigkeiten verbunden ist: Sofern man nicht präzise den Titel des Artikels eingibt, wird man entweder nicht fündig oder bekommt eine Trefferliste präsentiert, die - wie beim obigen Beispiel »Mose - alle Artikel nennt, die das Stichwort im Namen haben. Demnach ist nicht einmal bei präziser Eingabe des Artikeltitels »Mose« eine Direktverlinkung gegeben.

Schließlich ist es auf der Startseite ebenfalls möglich, in einem Themenregister (https://www. bibelwissenschaft.de/wibilex/thematisches-verzeichnis [22.04.2020]) nach bestimmten Sachgebieten und den dazu vorhandenen Artikel zu recherchieren. Hier ist zu beachten, dass man sich ob der Erscheinungsform des Mauszeigers nicht irritieren lassen darf: Er nimmt die weder die Standardform (Pfeil) noch die Zeigerform (Zeigefinger) an, sondern wird in der Textform (Balken) dargestellt.

Die Artikel als solche besitzen einen grundsätzlich gleichen Aufbau, unterscheiden sich aber in einiger Hinsicht auch strukturell im Detail. So ist in der Kopfzeile immer zunächst der*die Autor*in (im Fall des Beispiels »Mose« handelt es sich um J.Ch. Gertz) sowie Monat und Jahr der Abfassung (»Mose«: August 2008, Aktualisierung von 2020) genannt, wobei weiterführende Informationen zur Person und ihren Artikeln verlinkt sind. Außerdem ist es in der Kopfzeile möglich, den Artikel als PDF-Dokument herunterzuladen oder durch einen zitierfähigen Link permanent auf den Artikel zu verweisen.

So muss der Artikel »Mose « nicht unter der im Browser angezeigten, ausgesprochen langen Adresse https://www.bibelwissenschaft.de/wibilex/das-bibellexikon/sachwort/anzeigen/details/mose/ch/9f02bcba0c82b17ab9512a241d916033/ zitiert werden, sondern kann schlank mit https://www.bibelwissenschaft.de/stichwort/28069/ (beide 12.06.2020) angegeben werden.

Jeder Artikel schließt mit einem Literatur- und (sofern nötig) einem Abbildungsverzeichnis. Letzteres deutet auf einen weiteren hilfreichen Aspekt des WiBiLex 
hin: Viele Artikel sind mit teilweise lediglich illustrierenden, teilweise aber auch inhaltlich hilfreichen Abbildungen wie z.B. Kartenmaterial versehen, was direkt in den jeweiligen Artikel eingebettet ist und nicht erst in einem eigenen Tafelteil oder gar in anderen Büchern gesucht werden muss.

Kategorial vergleichbare Artikel sind häufig ähnlich aufgebaut, wie man im Vergleich mit E. Gaß, »Bileam (AT)« gut sehen kann: In beiden Fällen wird zunächst der jeweilige Eigenname in Herkunft und Bedeutung behandelt, im weiteren Verlauf geht es um die biblische Bezeugung, um die außerbiblische Bezeugung bzw. um Fragen der Historizität - im Fall des Mose werden hier viele wichtige Hinweise z.B. auf die Entstehung des Pentateuch, das ramessidische Ägypten oder die Geburtslegende des Sargon von Akkad gegeben, was Personen ohne Vorkenntnisse diverse Vertiefungsmöglichkeiten bietet - und schließlich um die Rezeption der jeweiligen Person in der Religions-, Kulturund Kunstgeschichte.

Der Rezeption biblischer Themen und Figuren in Bildender Kunst, Musik und Literatur ist mit »Die Bibel in der Kunst $(\mathrm{BiKu})$ «bzw. »Bible in the Arts« $(\mathrm{BiA})$ darüber hinaus eine eigene Seite auf www.bibelwissenschaft.de gewidmet. Anders als beim WiBiLex handelt es sich dabei nicht um ein Lexikon, sondern um bislang drei Jahrgänge von 2017 mit rezeptionshistorischen Fachaufsätzen, die allesamt als PDF heruntergeladen werden können und von Salomo-Dramen des 17. Jh. bis zum Leviathan im Film ein breites Spektrum abbilden.

Als drittes kurzes Beispiel soll an dieser Stelle K. Weingart, »Volk (AT)« von 2016 dienen. Hier werden nicht nur im Dreischritt »Terminologie - Volk Israel - Israel und die Völker« philologische Argumentation fachlich angemessen auch für Personen ohne Hebräischkenntnisse nachvollziehbar aufbereitet, sondern es findet sich auch der Verweis auf Debatten zum Volksbegriff außerhalb der Theologie. Eine vergleichbare Kombination aus Aktualität, fachlicher Tiefe und breiter Zielgruppe dürfte es - wie diese drei Artikel exemplarisch zeigen - jenseits des WiBiLex kaum geben, obgleich sich die Qualität und vor allem der Umfang der einzelnen Artikel teilweise merklich unterscheiden.

Neben dem zumeist jungen Entstehungsdatum der einzelnen Artikel ist die leichte Recherche eine weitere Stärke: Es können einerseits andere vorhandene Lexikonartikel verlinkt werden, die dadurch leicht zugänglich sind; andererseits können aber auch biblische Verweise direkt nachgelesen werden, da einzelne Bibelstellen angeklickt und die Stelle samt Nahkontext nach Luther 1984 gelesen werden.

Mit dem WiBiLex ist ein kostenfreies, online allgemein zugängliches Fachangebot gelungen, das konkrete Recherchemöglichkeiten von zeitgenössischen Fachautor*innen bietet. Dabei ist das Lexikon in seinen Vor- und Nachteilen von analogen Lexika und Fachpublikationen deutlich abzugrenzen, deren inhaltliche Tiefe und thematische Breite es nicht immer erreichen kann. Es zielt auf den direkten Gebrauch durch ein breiteres Fachpublikum unabhängig von einer größeren Bibliothek. Ein kleines Manko stellt die erwähnte lange Ladezeit 
einzelner Artikel dar; außerdem fällt die Qualität sowie der Umfang einzelner Beiträge erwartbar unterschiedlich aus. In jedem Fall ist den Herausgeber*innen und der Deutschen Bibelgesellschaft für dieses höchst nützliche Hilfsmittel sehr zu danken, das mittlerweile eine vollauf berechtigte breite Anerkennung genießt.

\section{Neues Testament}

Moisés Mayordomo

WiBiLex hat zunächst als ein rein alttestamentliches Projekt begonnen. Die folgerichtige Erweiterung auf den zweiten Teil des christlichen Kanons erfolgte erst einige Jahre später. Beide Projekte arbeiten weitgehend parallel, eine gesamtredaktionelle Leitung fehlt. Das hat zur Folge, dass Beiträge zu gleichen Lemmata nicht aufeinander bezogen sind. Die Artikel »Homosexualität « im Alten Testament (von M. Zehnder) und im Neuen Testament (von S. Scholz) lassen z.B. zwei sehr unterschiedliche Bewertungen erkennen, die gerade angesichts der Aktualität des Themas ein interessantes Problem im Rahmen eines Lexikons anzeigen. Zehnder greift zu Beginn zwar den Gedanken auf, dass der Terminus »Homosexualität « anachronistisch sein könnte, lässt aber nach der Besprechung der einschlägigen Texte und Kontexte am Ende seines Artikels keinen Zweifel daran, dass nach Gen 1-2 die Vervollständigung des Menschen nur »durch einen zweiten Menschen vom anderen Geschlecht « möglich ist. Dass der Autor einen Artikel zu Homosexualität überhaupt mit einem Ausblick auf Gen 1-2 beendet und daraus ein essentialistisches Verständnis sexueller Identität ableitet, macht deutlich, dass es hier um mehr als »Sachinformation « geht; es geht zugleich um einen lexikographischen Eingriff in Gegenwartsdiskurse. Der Artikel von S. Scholz hingegen reflektiert gleich zu Beginn über das Problem der unterschiedlichen Diskursräume und nimmt sich der kulturhistorischen Frage nach anthropologischen Konstanten direkt an. Indem Scholz nicht nur historische und philologische Informationen bietet, sondern zugleich die kulturellen und hermeneutischen Probleme mit bedenkt, bietet er einen viel weiteren $\mathrm{Ab}$ wägungsspielraum als Zehnder. Beide Artikel könnten in der vorliegenden Form kaum in einem Bibellexikon, das eine einheitliche redaktionelle Strategie verfolgt, nacheinander stehen. Das Interessante am virtuellen Raum jedoch ist, dass es kein »Nacheinander « mehr gibt. Daher wird die fehlende inhaltliche Vernetzung der Lemmata im konkreten Gebrauch weniger störend wirken.

Das angesprochene Beispiel zeigt allerdings noch mehr auf: Es ist ein Zeichen für die Publikumsausrichtung dieses Lexikons. Anders als die großen Lexikonprojekte der letzten Jahrzehnte (Lexikon für Theologie und Kirche 3. Aufl., Religion in Geschichte und Gegenwart 4. Aufl., Theologische Realenzyklopädie, Anchor Bible Dictionary und das laufende Grossunternehmen Encyclopedia of the Bible and Its Reception) richtet sich WiBiLex an Studierende mit Grundwissen, interessierte Bildungsmenschen, Pfarrerinnen, Katecheten, Lehrpersonen, usw. Das hat nicht nur auf die sprachliche Präsentation Einfluss, sondern 
eröffnet auch die Möglichkeit, über den aktuellen Bezug eines Stichwortes zu reflektieren.

Es wäre reizvoll, über die Frage der Publikumsausrichtung hinaus WiBiLex mit anderen lexikographischen Projekten der Theologie zu vergleichen. Man wird schwerlich sagen können, dass ein Internet-Artikel per se dem Medium des gedruckten Wortes qualitativ unterlegen ist. Artikel wie etwa »Antipas (Herodes)« (Ch. Böttrich), »Römerbrief« (F.-W. Horn) oder »Sexualität« (W. Loader) würden jedem gedruckten Lexikon zur Ehre gereichen. Dennoch fehlt dem virtuellen Lexikon das Volumen und Gewicht - und damit auch die kulturgeschichtliche Gewichtigkeit - eines mehrbändigen Lexikons. Diese Feststellung ist jedoch bereits Ausdruck der verblassenden Nostalgie all jener, die im Studium noch mit Ehrfurcht einen Band der Theologischen Realenzyklopädie oder des Reallexikons für Antike und Christentum aus den Regalen gegriffen haben. Denn mit Blick auf die medialen Umwälzungen der digitalen Welt muss man zugeben, dass die Produktion und Präsentation von Texten im Internet gerade für das Format eines Lexikons besonders gut geeignet sind. Die Verknüpfung über Hyperlinks, mit der wir mittlerweile fast schon intuitiv vertraut sind, die Verbindung von Text und Bild und die simplen Formen des Um- und Weiterschreibens gewährleisten Information, Aktualität und Vernetzung in einem Ausmaß, wie es ein gedrucktes Lexikon nicht zu leisten vermag. In welche Richtung das führen wird, zeigt sich auf der Einstiegsseite www.bibelwissenschaft.de (08.08.2020): Neben WiBiLex gibt es eine Auswahl der wichtigsten Bibeln im Original und in Übersetzung, ein »Wissenschaftlich-Religionspädagogisches Lexikon«(WiReLex), ein eJournal »Die Bibel in der Kunst«, eine Online-Version der Bibelkunden von M. Rösel (AT) und K.-M. Bull (NT) und (im Anfangsstadium) einen Bibelkommentar. Ein derart anwachsender Hypertext zum Bibelstudium kann in gedruckter Form nicht existieren. Wir mögen den Geruch und das Gewicht der alten Lexika vermissen, aber die Zukunft des Lexikons gehört dem Internet.

\section{Digital theology \\ Systematisch-theologische Perspektiven}

auf ein entstehendes Forschungsfeld Frederike van Oorschot

Jonas Bedford-Strohm/Florian Höhne/Julian Zeyher-Quattlender (Hg.), Digitaler Strukturwandel der Öffentlichkeit. Ethik und politische Partizipation in interdisziplinärer Perspektive (Kommunikations- und Medienethik 10), Nomos Baden-Baden 2019, 234 S. - Heidi A. Campbell/ Stephen Garner/William Dyrness/Robert Johnston, Networked Theology. Negotiating Faith in Digital Culture (Engaging Culture), Baker Academic Grand Rapids (Michigan) 2016, X + 175 S. - Johanna Haberer, Digitale Theologie. Gott und die Medienrevolution der Gegenwart, 